\title{
Hankel, Toeplitz, and Hermitian-Toeplitz Determinants for Certain Close-to-convex Functions
}

\author{
Vasudevarao Allu, Adam Lecko@ and Derek K. Thomas
}

\begin{abstract}
Let $f$ be analytic in $\mathbb{D}=\{z \in \mathbb{C}:|z|<1\}$, and be given by $f(z)=z+\sum_{n=2}^{\infty} a_{n} z^{n}$. We give sharp bounds for the second Hankel determinant, some Toeplitz, and some Hermitian-Toeplitz determinants of functions in the class of Ozaki close-to-convex functions, together with a sharp bound for the Zalcman functional $J_{2,3}(f)$.
\end{abstract}

Mathematics Subject Classification. Primary 30C45, 30C55.

Keywords. Univalent functions, Ozaki close-to-convex functions, Hankel, Toeplitz, Hermitian-Toeplitz, determinants, coefficients, Zalcman functional.

\section{Introduction and definitions}

Let $\mathcal{A}$ denote the class of functions $f$ analytic in the unit disk $\mathbb{D}:=\{z \in \mathbb{C}$ : $|z|<1\}$ with Taylor series

$$
f(z)=\sum_{n=1}^{\infty} a_{n} z^{n}, \quad a_{1}:=1 .
$$

Let $\mathcal{S}$ be the subclass of $\mathcal{A}$, consisting of univalent (i.e., one-to-one) functions. A function $f \in \mathcal{A}$ is called starlike (with respect to the origin) if $f(\mathbb{D})$ is starlike with respect to the origin, and convex if $f(\mathbb{D})$ is convex. Let $\mathcal{S}^{*}(\alpha)$ and $\mathcal{C}(\alpha)$ denote, respectively, the classes of starlike and convex functions of order $\alpha$ for $0 \leq \alpha<1$ in $\mathcal{S}$. It is well known that a function $f \in \mathcal{A}$ belongs to $\mathcal{S}^{*}(\alpha)$ if, and only if, $\operatorname{Re}\left(z f^{\prime}(z) / f(z)\right)>\alpha$ for $z \in \mathbb{D}$, and that $f \in \mathcal{C}(\alpha)$ if, and only if, $\operatorname{Re}\left(1+z f^{\prime \prime}(z) / f^{\prime}(z)\right)>\alpha$. We write $\mathcal{S}^{*}(0)=: \mathcal{S}^{*}$, and $\mathcal{C}(0)=: \mathcal{C}$.

Similarly, a function $f \in \mathcal{A}$ belongs to $\mathcal{K}$, the class of close-to-convex functions if, and only if, there exists $g \in \mathcal{S}^{*}$, such that $\operatorname{Re}\left[\mathrm{e}^{\mathrm{i} \tau}\left(z f^{\prime}(z) / g(z)\right)\right]>$ 0 for $z \in \mathbb{D}$, and $\tau \in(-\pi / 2, \pi / 2)$, so that $\mathcal{C} \subset \mathcal{S}^{*} \subset \mathcal{K} \subset \mathcal{S}$. When $\tau:=0$, the resulting subclass of close-to-convex functions is denoted by $\mathcal{K}_{0}$. 
Although the class $\mathcal{K}$ was first formally introduced by Kaplan in 1952 [14], in 1941, Ozaki [20] showed that a function in $\mathcal{A}$ is univalent if it satisfies the condition

$$
\operatorname{Re}\left(1+\frac{z f^{\prime \prime}(z)}{f^{\prime}(z)}\right)>-\frac{1}{2}, \quad z \in \mathbb{D} .
$$

It follows from the original definition of Kaplan [14], that functions satisfying (2) are close-to-convex, and therefore members of $\mathcal{S}$.

Robertson [22] considered the following generalization to (2) for $-1 / 2<$ $\lambda \leq 1 / 2$.

Definition 1.1. Let $f \in \mathcal{A}$ and be locally univalent for $z \in \mathbb{D}$, and $-1 / 2<$ $\lambda \leq 1$. Then, $f \in \mathcal{F}(\lambda)$ if and only if

$$
\operatorname{Re}\left(1+\frac{z f^{\prime \prime}(z)}{f^{\prime}(z)}\right)>\frac{1}{2}-\lambda, \quad z \in \mathbb{D} .
$$

Clearly, when $-1 / 2<\lambda \leq 1 / 2$ functions defined by (3) provide a subset of $\mathcal{C}$, with $\mathcal{F}(1 / 2)=\mathcal{C}$, and since $1 / 2-\lambda \geq-1 / 2$ when $\lambda \leq 1$, functions in $\mathcal{F}(\lambda)$ are close-to-convex when $1 / 2 \leq \lambda \leq 1$. Although functions in $\mathcal{F}(\lambda)$ are close-to-convex when $1 / 2 \leq \lambda \leq 1$, Pfaltzgraff, Reade, and Umezawa [21] showed that the classes $\mathcal{F}(\lambda)$ contain non-starlike functions for all $1 / 2<\lambda \leq$ 1. Moreover, Umezawa [24] proved that functions in $\mathcal{F}(1)$ are convex in one direction.

We shall call members of $f \in \mathcal{F}(\lambda)$ when $1 / 2 \leq \lambda \leq 1$, Ozaki close-toconvex functions, and denote this class by $\mathcal{F}_{O}(\lambda)$, noting that $\mathcal{F}_{O}(1)$ consists of the functions satisfying (2).

We note that in contrast to the definition of $\mathcal{K}$, the definition of $\mathcal{F}(\lambda)$ does not involve an independent starlike functions $g$, but as was shown by Umezawa [24], members of $\mathcal{F}(1)$ have coefficients which grow at the same rate as those in $\mathcal{K}$.

The following sharp bound for $\left|a_{n}\right|$ when $f \in \mathcal{F}_{O}(\lambda)$ was also obtained by Robertson [22] for the range $\lambda \in(-1 / 2,1 / 2]$ and Brickman, Hallenbeck, MacGregor, and Wilken in [6, Theorem 4] for $\lambda>-1 / 2$.

Theorem 1.1. Let $f \in \mathcal{F}(\lambda), \lambda>-1 / 2$, and be given by (1). Then, for $n \geq 2$

$$
\left|a_{n}\right| \leq \frac{1}{n !} \prod_{k=2}^{n}(k+2 \lambda-1) .
$$

The inequality is sharp when

$$
f(z)=f_{\lambda}(z):=\frac{1}{2 \lambda}\left(\frac{1}{(1-z)^{2 \lambda}}-1\right), \quad z \in \mathbb{D} .
$$

Other related results for $f \in \mathcal{F}_{O}(\lambda)$ were also obtained in [3], but the problem of finding the sharp bound for the second Hankel determinant $\mid a_{2} a_{4}-$ $a_{3}^{2} \mid$ was not considered.

In this paper, we will give the sharp bound for the second Hankel determinant, together with sharp bounds for various Toeplitz and HermitianToeplitz determinants defined below, whose elements are coefficients of functions in $\mathcal{F}_{O}(\lambda)$. 
We first give definitions of Hankel, Toeplitz, and Hermitian-Toeplitz determinants when $f \in \mathcal{A}$.

Definition 1.2. Let $f \in \mathcal{A}$, and be given by (1). Then, the $q$ th Hankel determinant is defined for $q \geq 1$ and $n \geq 0$ by

$$
H_{q}(n)(f):=\left|\begin{array}{cccc}
a_{n} & a_{n+1} & \ldots & a_{n+q-1} \\
a_{n+1} & a_{n+2} & \ldots & a_{n+q} \\
\vdots & \vdots & \vdots & \vdots \\
a_{n+q-1} & a_{n+q} & \ldots & a_{n+2 q-2}
\end{array}\right| .
$$

In particular,

$$
H_{2}(2)(f)=a_{2} a_{4}-a_{3}^{2} .
$$

Definition 1.3. Let $f \in \mathcal{A}$, and be given by (1). Then, the $q$ th Toeplitz determinant is defined for $q \geq 1$ and $n \geq 0$ by

$$
T_{q}(n)(f):=\left|\begin{array}{cccc}
a_{n} & a_{n+1} & \ldots & a_{n+q-1} \\
a_{n+1} & a_{n} & \ldots & a_{n+q-2} \\
\vdots & \vdots & \vdots & \vdots \\
a_{n+q-1} & a_{n+q-2} & \ldots & a_{n}
\end{array}\right| .
$$

In particular

$$
T_{3}(1)(f)=1-2 a_{2}^{2}+2 a_{2}^{2} a_{3}-a_{3}^{2}
$$

and

$$
T_{3}(2)(f)=a_{2}^{3}-2 a_{2} a_{3}^{2}+2 a_{3}^{2} a_{4}-a_{2} a_{4}^{2} .
$$

Definition 1.4. Let $f \in \mathcal{A}$, and be given by (1). Then, for $q \geq 1$ and $n \geq 0$, define

$$
T_{q, n}(f):=\left|\begin{array}{cccc}
a_{n} & a_{n+1} & \ldots & a_{n+q-1} \\
\bar{a}_{n+1} & a_{n} & \ldots & a_{n+q-2} \\
\vdots & \vdots & \vdots & \vdots \\
\bar{a}_{n+q-1} & \bar{a}_{n+q-2} & \ldots & a_{n}
\end{array}\right|,
$$

where $\bar{a}_{k}:=\overline{a_{k}}$. When $a_{n}$ is a real number, $T_{q, n}(f)$ is $q$ th Hermitian-Toeplitz determinant.

In particular

$$
T_{3,1}(f)=1-2\left|a_{2}\right|^{2}+2 \operatorname{Re}\left(a_{2}^{2} \bar{a}_{3}\right)-\left|a_{3}\right|^{2} .
$$

Finding sharp bounds for the Hankel determinants of functions in $\mathcal{A}$ has been the subject of a great many papers in the recent years. In particular, many results are known concerning the second Hankel determinant $H_{2}(2)=a_{2} a_{4}-a_{3}^{2}$ when $f \in \mathcal{S}$ and its subclasses, and a summary of some of the more important results can be found in [23]. On the other hand, investigations concerning Toeplitz determinants were introduced only recently in [2]. Similarly, problems concerning Hermitian-Toeplitz determinants were first considered in [9].

We next discuss the Zalcman functional, its relationship with the Zalcman conjecture, and a generalization due to Ma [19]. 
In the early $70 \mathrm{~s}$, Lawrence Zalcman posed the conjecture that if $f \in \mathcal{S}$, and is given by (1), then

$$
\left|a_{n}^{2}-a_{2 n-1}\right| \leq(n-1)^{2} \quad \text { for } n \geq 2,
$$

with equality for the Koebe function $k(z)=z /(1-z)^{2}$ for $z \in \mathbb{D}$, or a rotation. This conjecture implies the celebrated Bieberbach conjecture $\left|a_{n}\right| \leq n$ for $f \in \mathcal{S}$ (see [7]). The elementary area theorem shows that the conjecture is true when $n=2$ (see [10]). Kruskal established the conjecture when $n=3$ (see [15]), and more recently for $n=4,5,6$ (see [16]). However, the Zalcman conjecture for $n>6$ remains an open problem.

The conjecture has been proved for several subclasses of $\mathcal{S}$, e.g., starlike, typically real, and close-to-convex functions [7,18], and it is known that the Zalcman conjecture is asymptotically true (see [12]). Recently, Abu Muhana et al. [1] proved the conjecture for the class $\mathcal{F}_{O}(1)$.

Relevant to this paper is Ma's generalization of the Zalcman functional $a_{n}^{2}-a_{2 n-1}$, defined as follows.

Definition 1.5. Let $f \in \mathcal{A}$, and be given by (1). For $m, n \in \mathbb{N} \backslash\{1\}$, let $J_{m, n}(f):=a_{m} a_{n}-a_{m+n-1}$, and in particular, $J_{2,3}(f)=a_{2} a_{3}-a_{4}$.

In [19], Ma conjectured that if $f \in \mathcal{S}$, then for $m, n \in \mathbb{N} \backslash\{1\}$

$$
\left|J_{m, n}(f)\right| \leq(n-1)(m-1),
$$

and established the conjecture when $f \in \mathcal{S}^{*}$, and if $f \in \mathcal{S}$, provided that the coefficients in (1) are real.

In a recent paper, Cho et al. [8] considered the case $m=2, n=3$, for a very wide set of functions in $\mathcal{A}$, obtaining sharp bounds for $\left|J_{2,3}(f)\right|$ in all cases, where we note that Theorem $4.1 \mathrm{C}$ provides a sharp bound for $\left|J_{2,3}(f)\right|$, when $f$ satisfies inequality $(3)$ for $\lambda \in(-1 / 2,1 / 2]$.

\section{Lemmas}

We will use the following results for functions $p \in \mathcal{P}$, the class of functions with positive real part in $\mathbb{D}$, given by

$$
p(z)=1+\sum_{n=1}^{\infty} p_{n} z^{n}
$$

and since we will be concerned primarily with the coefficients $a_{2}, a_{3}$, and $a_{4}$, we also need Lemma 2.4, which can easily be deduced from (1), (3), and (6).

Lemma 2.1. ([10]) Let $p \in \mathcal{P}$, and be given by (6), then $\left|p_{n}\right| \leq 2$, when $n \geq 2$. Also

$$
\left|p_{2}-\frac{\mu}{2} p_{1}^{2}\right| \leq \max \{2,2|\mu-1|\}= \begin{cases}2, & 0 \leq \mu \leq 2 \\ 2|\mu-1|, & \text { elsewhere }\end{cases}
$$

Lemma 2.2. ([11]) If $p \in \mathcal{P}$, and is given by (6), then for $\mu \in \mathbb{C}$, and $1 \leq k \leq$ $n-1$

$$
\left|p_{n}-\mu p_{k} p_{n-k}\right| \leq 2 \max \{1,|2 \mu-1|\}
$$


Lemma 2.3. ([17]) Suppose that $p \in \mathcal{P}$, with coefficients given by (6), and $p_{1} \geq 0$. Then, for some complex-valued $y$ with $|y| \leq 1$, and some complexvalued $\zeta$ with $|\zeta| \leq 1$

$$
\begin{aligned}
& 2 p_{2}=p_{1}^{2}+y\left(4-p_{1}^{2}\right), \\
& 4 p_{3}=p_{1}^{3}+2\left(4-p_{1}^{2}\right) p_{1} y-p_{1}\left(4-p_{1}^{2}\right) y^{2}+2\left(4-p_{1}^{2}\right)\left(1-|y|^{2}\right) \zeta .
\end{aligned}
$$

Lemma 2.4. Suppose $f \in \mathcal{F}_{0}(\lambda)$, and is given by (1). Then

$$
\begin{aligned}
& a_{2}=\frac{1}{4} p_{1}(1+2 \lambda), \\
& a_{3}=\frac{1}{12}(1+2 \lambda)\left(p_{2}+\frac{1}{2} p_{1}^{2}(1+2 \lambda)\right), \\
& a_{4}=\frac{1}{24}(1+2 \lambda)\left(p_{3}+\frac{3}{4} p_{1} p_{2}(1+2 \lambda)+\frac{1}{8} p_{1}^{3}(1+2 \lambda)^{2}\right),
\end{aligned}
$$

where $p_{1}, p_{2}$, and $p_{3}$ are given by $(6)$.

\section{The second Hankel determinant $H_{2}(2)(f)$}

Theorem 3.1. If $f \in \mathcal{F}_{O}(\lambda), 1 / 2 \leq \lambda \leq 1$, then

$$
\left|H_{2}(2)(f)\right| \leq \frac{(1+2 \lambda)^{2}(17-10 \lambda)}{192(3-2 \lambda)} .
$$

The inequality is sharp.

Proof. First note that from (3), we can write

$$
1+\frac{z f^{\prime \prime}(z)}{f^{\prime}(z)}=\left(\frac{1}{2}+\lambda\right) p(z)+\frac{1}{2}-\lambda, \quad z \in \mathbb{D} .
$$

Thus, from Lemma 2.4, we have

$$
\begin{aligned}
a_{2} a_{4}-a_{3}^{2}= & -\frac{(1+2 \lambda)^{4} p_{1}^{4}}{2304}+\frac{(1+2 \lambda)^{3} p_{1}^{2} p_{2}}{1152} \\
& -\frac{1}{144}(1+2 \lambda)^{2} p_{2}^{2}+\frac{1}{96}(1+2 \lambda)^{2} p_{1} p_{3} .
\end{aligned}
$$

Noting that both the class $\mathcal{F}_{O}(\lambda)$ and the functional $H_{2}(2)(f)$ are rotationally invariant, we now use Lemma 2.3 to express the coefficients $p_{3}$ and $p_{2}$ in terms of $p_{1}$, and write $t:=p_{1}$ to obtain with $0 \leq t \leq 2$

$$
\begin{aligned}
a_{2} a_{4}-a_{3}^{2}= & -\frac{(1+\lambda)(2 \lambda-1)(1+2 \lambda)^{2} t^{4}}{1152}+\frac{(1+2 \lambda)^{2}(5+2 \lambda) t^{2}\left(4-t^{2}\right) y}{2304} \\
& -\frac{1}{384}(1+2 \lambda)^{2} t^{2}\left(4-t^{2}\right) y^{2}-\frac{1}{576}(1+2 \lambda)^{2}\left(4-t^{2}\right)^{2} y^{2} \\
& +\frac{1}{192}(1+2 \lambda)^{2} t\left(4-t^{2}\right)\left(1-|y|^{2}\right) \zeta
\end{aligned}
$$


We now take the modulus to obtain

$$
\begin{aligned}
\left|H_{2}(2)(f)\right| \leq & \frac{(1+\lambda)(2 \lambda-1)(1+2 \lambda)^{2} t^{4}}{1152}+\frac{(1+2 \lambda)^{2}(5+2 \lambda) t^{2}\left(4-t^{2}\right)|y|}{2304} \\
& +\frac{1}{384}(1+2 \lambda)^{2} t^{2}\left(4-t^{2}\right)|y|^{2}+\frac{1}{576}(1+2 \lambda)^{2}\left(4-t^{2}\right)^{2}|y|^{2} \\
& +\frac{1}{192}(1+2 \lambda)^{2} t\left(4-t^{2}\right)\left(1-|y|^{2}\right) \\
= & \frac{(1+\lambda)(2 \lambda-1)(1+2 \lambda)^{2} t^{4}}{1152}+\frac{(1+2 \lambda)^{2}(5+2 \lambda) t^{2}\left(4-t^{2}\right)}{2304}|y| \\
& +\frac{1}{1152}(1+2 \lambda)^{2}\left(4-t^{2}\right)(4-t)(2-t)|y|^{2} \\
& +\frac{1}{192}(1+2 \lambda)^{2} t\left(4-t^{2}\right)=: \phi(t,|y|) .
\end{aligned}
$$

For $t=2$, we have

$$
\left|H_{2}(2)(f)\right|=\frac{1}{72}(1+\lambda)(2 \lambda-1)(1+2 \lambda)^{2} \leq \frac{1}{36}(1+2 \lambda)^{2} .
$$

For $0 \leq t<2$, the function $[0,1] \ni|y| \mapsto \phi(t,|y|)$ is easily seen to be increasing, so

$$
\begin{aligned}
\left|H_{2}(2)(f)\right| & \leq \phi(t,|y|) \leq \phi(t, 1) \\
& =\frac{(1+2 \lambda)^{2}}{2304}\left(\left(4 \lambda^{2}-9\right) t^{4}+4(2 \lambda+3) t^{2}+64\right) .
\end{aligned}
$$

Thus, the function $[0,2) \ni t \mapsto \phi(t, 1)$ has critical points at

$$
t=0 \text { and } t=\frac{\sqrt{2}}{\sqrt{3-2 \lambda}}=: t_{0}
$$

with values $\frac{1}{36}(1+2 \lambda)^{2}$, and $\frac{(1+2 \lambda)^{2}(17-10 \lambda)}{192(3-2 \lambda)}$, respectively, and since

$$
\frac{1}{36}(1+2 \lambda)^{2} \leq \frac{(1+2 \lambda)^{2}(17-10 \lambda)}{192(3-2 \lambda)}
$$

when $1 / 2 \leq \lambda \leq 1$, the theorem is proved.

To see that the inequality is sharp, take a function

$$
p(z)=\frac{1-z^{2}}{1-t_{0} z+z^{2}}, \quad z \in \mathbb{D}
$$

for which

$$
p_{1}=\frac{\sqrt{2}}{\sqrt{3-2 \lambda}}, \quad p_{2}=-\frac{4(1-\lambda)}{3-2 \lambda}, \quad \text { and } \quad p_{3}=\frac{\sqrt{2}(-7+6 \lambda)}{(3-2 \lambda)^{3 / 2}} .
$$

Choosing $\lambda=1 / 2$, and $\lambda=1$, we deduce the following sharp inequalities. 
Corollary 3.2. If $f \in \mathcal{C}$, then

$$
\left|H_{2}(2)(f)\right| \leq \frac{1}{8}
$$

proved in [13], and if $f \in \mathcal{F}_{O}(1)$, i.e., satisfying (2), then

$$
\left|H_{2}(2)(f)\right| \leq \frac{21}{64}
$$

proved in [5].

\section{Toeplitz determinants}

In this section, we extend the results in [2] for $f \in \mathcal{C}$ to $f \in \mathcal{F}_{O}(\lambda)$.

We first define the function $f_{1} \in \mathcal{F}_{O}(\lambda)$ for $z \in \mathbb{D}$, which serves as the extreme function for all the following results:

$$
\begin{aligned}
f_{1}(z):= & \frac{1}{2 \mathrm{i} \lambda}\left(\frac{1}{(1-\mathrm{i} z)^{2 \lambda}}-1\right)=z+\sum_{n=2}^{\infty}(\mathrm{i})^{n-1} d_{n} z^{n} \\
= & z+\frac{\mathrm{i}}{2}(1+2 \lambda) z^{2}-\frac{1}{3}(1+\lambda)(1+2 \lambda) z^{3} \\
& -\frac{\mathrm{i}}{12}(1+\lambda)(1+2 \lambda)(3+2 \lambda) z^{4}+\ldots, \quad z \in \mathbb{D},
\end{aligned}
$$

where

$$
d_{n}:=\frac{1}{n !} \prod_{k=2}^{n}(k+2 \lambda-1) .
$$

Theorem 4.1. If $f \in \mathcal{F}_{O}(\lambda), 1 / 2 \leq \lambda \leq 1$, then for $n \geq 2$

$$
\left|T_{2}(n)(f)\right| \leq\left[\frac{1}{(n+1) !} \prod_{k=2}^{n}(k+2 \lambda-1)\right]^{2}\left[(n+2 \lambda)^{2}+(n+1)^{2}\right] .
$$

The inequality is sharp when $f=f_{1}$.

Proof. We simply note that

$$
\left|T_{2}(n)(f)\right|=\left|a_{n}^{2}-a_{n+1}^{2}\right| \leq\left|a_{n}\right|^{2}+\left|a_{n+1}\right|^{2},
$$

and apply (4).

To see that this is sharp, recall that

$$
f_{1}(z)=z+\sum_{n=2}^{\infty}(\mathrm{i})^{n-1} d_{n} z^{n}, \quad z \in \mathbb{D} .
$$

Then

$$
\left|\left(\mathrm{i}^{n-1} d_{n}\right)^{2}-\left(\mathrm{i}^{n} d_{n+1}\right)^{2}\right|=d_{n}^{2}+d_{n+1}^{2},
$$

which, using (14), shows that the inequality for $T_{2}(n)(f)$ is sharp.

Theorem 4.2. If $f \in \mathcal{F}_{O}(\lambda), 1 / 2 \leq \lambda \leq 1$, then

$$
\left|T_{3}(1)(f)\right| \leq \frac{1}{18}\left(4+3 \lambda+2 \lambda^{2}\right)\left(7+6 \lambda+8 \lambda^{2}\right) .
$$

The inequality is sharp when $f=f_{1}$. 
Proof. We first note that

$$
\begin{aligned}
\left|T_{3}(1)(f)\right| & =\left|1-2 a_{2}^{2}+2 a_{2}^{2} a_{3}-a_{3}^{2}\right| \\
& \leq 1+2\left|a_{2}\right|^{2}+\left|a_{3}\right|\left|a_{3}-2 a_{2}^{2}\right| \\
& \leq 1+\frac{1}{2}(1+2 \lambda)^{2}+\frac{1}{3}(1+\lambda)(1+2 \lambda)\left|a_{3}-2 a_{2}^{2}\right|,
\end{aligned}
$$

where we have used Lemmas 2.1 and 2.4.

It therefore remains to estimate $\left|a_{3}-2 a_{2}^{2}\right|$.

Note first that

$$
a_{3}-2 a_{2}^{2}=\frac{1}{12}(1+2 \lambda)\left(p_{2}-p_{1}^{2}(1+2 \lambda)\right) .
$$

Thus, taking $\mu=2(1+2 \lambda)$, we deduce from Lemma 2.1 that

$$
\left|a_{3}-2 a_{2}^{2}\right| \leq 2(1+4 \lambda),
$$

and so, from (15), we obtain

$$
\begin{aligned}
\left|T_{3}(1)(f)\right| & \left.\leq 1+\frac{1}{2}(1+2 \lambda)^{2}+\frac{2}{3}(1+\lambda)(1+2 \lambda)(1+4 \lambda)\right) \\
& =\frac{1}{18}\left(4+3 \lambda+2 \lambda^{2}\right)\left(7+6 \lambda+8 \lambda^{2}\right) .
\end{aligned}
$$

For equality in Theorem 4.2, we choose $f=f_{1}$ defined in (13).

Theorem 4.3. If $f \in \mathcal{F}_{O}(\lambda), 1 / 2 \leq \lambda \leq 1$, then

$$
\left|T_{3}(2)(f)\right| \leq \frac{1}{864}(1+2 \lambda)^{3}\left(9+5 \lambda+2 \lambda^{2}\right)\left(25+17 \lambda+10 \lambda^{2}\right) .
$$

The inequality is sharp when $f=f_{1}$.

Proof. We first note that

$$
\left|T_{3}(2)(f)\right|=\left|\left(a_{2}-a_{4}\right)\left(a_{2}^{2}-2 a_{3}^{2}+a_{2} a_{4}\right)\right|,
$$

and since $\left|a_{2}-a_{4}\right| \leq\left|a_{2}\right|+\left|a_{4}\right|$, from Theorem 1.1, we have

$$
\left|a_{2}-a_{4}\right| \leq \frac{1}{12}(1+2 \lambda)\left(9+5 \lambda+2 \lambda^{2}\right) .
$$

Thus, it remains to estimate $\left|a_{2}^{2}-2 a_{3}^{2}+a_{2} a_{4}\right|$.

Using Lemma 2.4, we obtain

$$
\begin{aligned}
a_{2}^{2}-2 a_{3}^{2}+a_{2} a_{4}= & \frac{1}{16} p_{1}^{2}(1+2 \lambda)^{2}-\frac{1}{72} p_{2}^{2}(1+2 \lambda)^{2}+\frac{1}{96} p_{1} p_{3}(1+2 \lambda)^{2} \\
& -\frac{7}{1152} p_{1}^{2} p_{2}(1+2 \lambda)^{3}-\frac{5}{2304} p_{1}^{4}(1+2 \lambda)^{4}
\end{aligned}
$$

Taking the modulus, we obtain

$$
\begin{aligned}
\left|a_{2}^{2}-2 a_{3}^{2}+a_{2} a_{4}\right| \leq & \frac{1}{144}(1+2 \lambda)^{2}\left(44+5(1+2 \lambda)^{2}\right) \\
& +\frac{1}{48}(1+2 \lambda)^{2}\left|p_{3}-\frac{7}{12}(1+2 \lambda) p_{1} p_{2}\right| .
\end{aligned}
$$

Since Lemma 2.2 gives

$$
\left|p_{3}-\frac{7}{12}(1+2 \lambda) p_{1} p_{2}\right| \leq \frac{1}{3}(1+14 \lambda),
$$


we obtain

$$
\left|a_{2}^{2}-2 a_{3}^{2}+a_{2} a_{4}\right| \leq \frac{1}{72}(1+2 \lambda)^{2}\left(25+17 \lambda+10 \lambda^{2}\right),
$$

and so

$$
\left|T_{3}(2)(f)\right| \leq \frac{1}{864}(1+2 \lambda)^{3}\left(9+5 \lambda+2 \lambda^{2}\right)\left(25+17 \lambda+10 \lambda^{2}\right)
$$

as required.

The inequality is sharp on again choosing $f=f_{1}$ defined in (13).

\section{Hermitian-Toeplitz determinants}

In this section, we compute sharp lower and upper bounds for

$$
T_{3,1}(f)=\left|\begin{array}{ccc}
1 & a_{2} & a_{3} \\
\bar{a}_{2} & 1 & a_{2} \\
\bar{a}_{3} & \bar{a}_{2} & 1
\end{array}\right|=2 \operatorname{Re}\left(a_{2}^{2} \bar{a}_{3}\right)-2\left|a_{2}\right|^{2}-\left|a_{3}\right|^{2}+1
$$

over the class $\mathcal{F}_{O}(\lambda)$.

Theorem 5.1. If $f \in \mathcal{F}_{O}(\lambda), 1 / 2 \leq \lambda \leq 1$, then

$$
T_{3,1}(f) \leq \begin{cases}1, & \lambda \in[1 / 2,(\sqrt{153}-5) / 8], \\ 1+\frac{1}{18}(1+2 \lambda)^{2}\left(4 \lambda^{2}+5 \lambda-8\right), & \lambda \in((\sqrt{153}-5) / 8,1],\end{cases}
$$

and

$$
T_{3,1}(f) \geq-\frac{(2 \lambda-1)^{2}(2 \lambda+5)^{2}}{64 \lambda(2 \lambda+3)} .
$$

Both inequalities are sharp.

Proof. First note that both $\mathcal{F}_{O}(\lambda)$ and $T_{3,1}(f)$ are rotationally invariant, and so, we can assume that $p_{1}=2 x$ for $x \in[0,1]$. Thus, using (16), Lemmas 2.3 and 2.4, we obtain

$$
\begin{aligned}
T_{3,1}(f)= & \frac{1}{24}(1+2 \lambda)^{3}\left[4(1+\lambda) x^{4}+2\left(1-x^{2}\right) x^{2} \operatorname{Re} y\right] \\
& -\frac{1}{36}(1+2 \lambda)^{2}\left[4(1+\lambda)^{2} x^{4}+4(1+\lambda)\left(1-x^{2}\right) x^{2} \operatorname{Re} y\right. \\
& \left.+\left(1-x^{2}\right)^{2}|y|^{2}\right]-\frac{1}{2}(1+2 \lambda)^{2} x^{2}+1 .
\end{aligned}
$$

for some complex $y$ with $|y| \leq 1$.

We consider two cases: $\mathbf{A}$, when $y \neq 0$, and $\mathbf{B}$, when $y=0$.

A. Suppose first that $y \neq 0$. Then, $y=|y| \mathrm{e}^{\mathrm{i} \varphi}$ with $|y| \in(0,1]$, and $\varphi \in[0,2 \pi)$.

Thus, setting $t:=x^{2}$, from (19), we get

$$
T_{3,1}(f)=G(t,|y|, \varphi),
$$


where

$$
\begin{aligned}
G(t, u, \varphi):= & \frac{1}{24}(1+2 \lambda)^{3}\left[4(1+\lambda) t^{2}+2(1-t) t u \cos \varphi\right] \\
& -\frac{1}{36}(1+2 \lambda)^{2}\left[4(1+\lambda)^{2} t^{2}+4(1+\lambda)(1-t) t u \cos \varphi\right. \\
& \left.+(1-t)^{2} u^{2}\right]-\frac{1}{2}(1+2 \lambda)^{2} t+1
\end{aligned}
$$

for $t \in[0,1], u \in[0,1], \varphi \in[0,2 \pi]$. Since

$$
\begin{aligned}
G(t, u, \varphi)= & -\frac{1}{36}(1+2 \lambda)^{2}(1-t)^{2} u^{2}+\frac{1}{36}(1+2 \lambda)^{2}(2 \lambda-1)(1-t) t u \cos \varphi \\
& +\frac{1}{18}(1+2 \lambda)^{2}(1+\lambda)(4 \lambda+1) t^{2}-\frac{1}{2}(1+2 \lambda)^{2} t+1,
\end{aligned}
$$

and $\lambda \in[1 / 2,1]$, we see that

$$
L(t, u) \leq G(t, u, \varphi) \leq P(t, u), \quad t \in[0,1], u \in[0,1], \varphi \in[0,2 \pi],
$$

where

$$
L(t, u):=G(t, u, \pi), \quad P(t, u):=G(t, u, 0) .
$$

I. We first discuss the inequality (17).

We have

$$
\begin{aligned}
P(t, u)= & -\frac{1}{36}(1+2 \lambda)^{2}(1-t)^{2} u^{2}+\frac{1}{36}(1+2 \lambda)^{2}(2 \lambda-1)(1-t) t u \\
& +\frac{1}{18}(1+2 \lambda)^{2}(1+\lambda)(4 \lambda+1) t^{2}-\frac{1}{2}(1+2 \lambda)^{2} t+1 .
\end{aligned}
$$

When $t=1$, i.e., $x=1$, then $p_{1}=2$, so

$$
P(1, u)=1+\frac{1}{18}(1+2 \lambda)^{2}\left(4 \lambda^{2}+5 \lambda-8\right), \quad u \in[0,1] .
$$

Assume next that $t \in[0,1)$, and let

$$
u_{w}:=\frac{(2 \lambda-1) t}{2(1-t)} .
$$

We consider two further cases.

Case 1. Suppose that $u_{w} \geq 1$, i.e., that $2 /(2 \lambda+1) \leq t<1$. Then

$$
P(t, u) \leq P(t, 1)=\frac{1}{18}(1+2 \lambda)^{4} t^{2}+\frac{1}{36}(1+2 \lambda)^{2}(2 \lambda-17) t-\frac{1}{36}(1+2 \lambda)^{2}+1 .
$$

Since

$$
\begin{aligned}
& t_{w}:=\frac{17-2 \lambda}{4(1+2 \lambda)^{2}}<\frac{2}{2 \lambda+1}, \\
& P(t, 1) \leq P(1,1)=1+\frac{1}{18}(1+2 \lambda)^{2}\left(4 \lambda^{2}+5 \lambda-8\right) .
\end{aligned}
$$

Case 2. Suppose that $0 \leq u_{w}<1$, i.e., that $0 \leq t<2 /(2 \lambda+1)$. Then

$$
P(t, u) \leq P\left(t, u_{w}\right)=\left(\frac{1}{4}(1+2 \lambda)^{2} t-1\right)^{2} \leq P\left(0, u_{w}\right)=1 .
$$


Noting now that

$$
P(1,1)=1+\frac{1}{18}(1+2 \lambda)^{2}\left(4 \lambda^{2}+5 \lambda-8\right)<1=P\left(0, u_{w}\right)
$$

if, and only if, $\lambda \in(1 / 2,(\sqrt{153}-5) / 8)$, combining (24) with (23) and (20), inequality (17) follows in the case when $y \neq 0$.

II. We next discuss the inequality (18).

We have

$$
\begin{aligned}
L(t, u)= & -\frac{1}{36}(1+2 \lambda)^{2}(1-t)^{2} u^{2}-\frac{1}{36}(1+2 \lambda)^{2}(2 \lambda-1)(1-t) t u \\
& +\frac{1}{18}(1+2 \lambda)^{2}(1+\lambda)(4 \lambda+1) t^{2}-\frac{1}{2}(1+2 \lambda)^{2} t+1
\end{aligned}
$$

for $t \in[0,1], u \in[0,1]$.

When $t=1$, i.e., $x=1$, so for $p_{1}=2$, we have

$$
L(1, u)=1+\frac{1}{18}(1+2 \lambda)^{2}\left(4 \lambda^{2}+5 \lambda-8\right), \quad u \in[0,1] .
$$

Assume next that $t \in[0,1)$ and let

$$
u_{w}^{\prime}:=-\frac{(2 \lambda-1) t}{2(1-t)} .
$$

Since $u_{w}^{\prime} \leq 0$, for $t \in[0,1)$, we have

$$
\begin{aligned}
L(t, u) & \geq L(t, 1) \\
& =\frac{1}{9} \lambda(1+2 \lambda)^{2}(3+2 \lambda) t^{2}-\frac{1}{36}(1+2 \lambda)^{2}(15+2 \lambda) t-\frac{1}{36}(1+2 \lambda)^{2}+1 .
\end{aligned}
$$

Let

$$
t_{w}^{\prime}:=\frac{2 \lambda+15}{8 \lambda(2 \lambda+3)} .
$$

It is easy to check, $t_{w}^{\prime}<1$, so

$$
L(t, 1) \geq L\left(t_{w}^{\prime}, 1\right)=-\frac{(2 \lambda-1)^{2}(2 \lambda+5)^{2}}{64 \lambda(2 \lambda+3)}, \quad t \in[0,1) .
$$

Note now that the inequality $L(1, u) \geq L\left(t_{w}^{\prime}, 1\right)$, i.e., the inequality

$$
1+\frac{1}{18}(1+2 \lambda)^{2}\left(4 \lambda^{2}+5 \lambda-8\right) \geq-\frac{(2 \lambda-1)^{2}(2 \lambda+5)^{2}}{64 \lambda(2 \lambda+3)}
$$

is equivalent to

$$
(2 \lambda-1)^{2}(2 \lambda+1)^{2}(8 \lambda+5)^{2} \geq 0,
$$

which clearly holds. Therefore, (26), together with (25), (20) and (23), proves (18) in this case also.

This completes the proof of the theorem in case $\mathbf{A}$.

B. Now, assume that $y=0$. Since

$$
T_{3,1}(f)=\frac{1}{18}(1+2 \lambda)^{2}(1+\lambda)(4 \lambda+1) t^{2}-\frac{1}{2}(1+2 \lambda)^{2} t+1=G(t, 0, \varphi)
$$

for $t \in[0,1]$ and $\varphi \in[0,2 \pi]$, and noting that (23) is true for $u=0$, by Parts I and II above, both inequalities (17) and (18) are true. 
It remains to discuss sharpness. When $\lambda \in(1 / 2,(\sqrt{153}-5) / 8]$, the identity function gives equality in (17).

When $\lambda \in((\sqrt{153}-5) / 8,1)$, the function $f$ given by (9) with

$$
p(z)=\frac{1+z}{1-z}, \quad z \in \mathbb{D},
$$

having coefficients

is extremal.

$$
a_{2}=\frac{1}{2}(1+2 \lambda), \quad a_{3}=\frac{1}{3}(1+2 \lambda)(1+\lambda)
$$

To see that the inequality (18) is sharp, take $f$ given by (9) with

$$
p(z)=\frac{1-z^{2}}{1-a z+z^{2}}, \quad z \in \mathbb{D}
$$

where $a:=2 \sqrt{t_{w}^{\prime}}$, for which

$$
p_{1}=2 \sqrt{\frac{2 \lambda+15}{8 \lambda(2 \lambda+3)}}, \quad p_{2}=\frac{-8 \lambda^{2}-10 \lambda+15}{2 \lambda(3+2 \lambda)} .
$$

Choosing $\lambda=1 / 2$, and $\lambda=1$, we deduce the following sharp inequalities.

Corollary 5.2. If $f \in \mathcal{C}$, then

$$
0 \leq T_{3,1}(f) \leq 1
$$

proved in $[9]$, and if $f \in \mathcal{F}_{O}(1)$, then

$$
-\frac{49}{320} \leq T_{3,1}(f) \leq \frac{3}{2} \text {. }
$$

\section{The functional $J_{2,3}(f)$}

We give the sharp upper bound for $\left|J_{2,3}(f)\right|$ when $f \in \mathcal{F}_{O}(\lambda)$.

Theorem 6.1. If $f \in \mathcal{F}_{O}(\lambda), 1 / 2 \leq \lambda \leq 1$, then

$$
\left|J_{2,3}(f)\right| \leq \frac{(1+2 \lambda)(7+2 \lambda)^{3 / 2}}{36 \sqrt{3\left(9-4 \lambda^{2}\right)}} .
$$

The inequality is sharp.

Proof. From Lemma 2.4, we have

$$
a_{2} a_{3}-a_{4}=\frac{(1+2 \lambda)^{3}}{192} p_{1}^{3}-\frac{(1+2 \lambda)^{2}}{96} p_{1} p_{2}-\frac{1+2 \lambda}{24} p_{3} .
$$

Noting that both $\mathcal{F}_{O}(\lambda)$ and $J_{2,3}(f)$ are rotationally invariant, we now use Lemma 2.3 to express the coefficients $p_{3}$ and $p_{2}$ in terms of $p_{1}$, and write $u:=p_{1}$ to obtain with $0 \leq u \leq 2$

$$
\begin{aligned}
a_{2} a_{3}-a_{4}= & \frac{1+2 \lambda}{192}\left[2(2 \lambda-1)(1+\lambda) u^{3}-(5+2 \lambda)\left(4-u^{2}\right) u y+2\left(4-u^{2}\right) u y^{2}\right. \\
& \left.-4\left(4-u^{2}\right)\left(1-|y|^{2}\right) \zeta\right] .
\end{aligned}
$$


Hence

$$
\begin{aligned}
\left|a_{2} a_{3}-a_{4}\right| \leq & \frac{1+2 \lambda}{192}\left[2(2 \lambda-1)(1+\lambda) u^{3}\right. \\
& \left.\left.+(5+2 \lambda)\left(4-u^{2}\right) u t+2\left(4-u^{2}\right) u t^{2}+4\left(4-u^{2}\right)\left(1-t^{2}\right)\right]\right] \\
= & : \psi(u, t),
\end{aligned}
$$

where $t:=|y| \in[0,1]$.

When $u=2$, since $192 \lambda^{6}+672 \lambda^{5}+36 \lambda^{4}-1516 \lambda^{3}+33 \lambda^{2}+996 \lambda+100>0$, when $1 / 2 \leq \lambda \leq 1$, we have

$$
\left|a_{2} a_{3}-a_{4}\right| \leq \frac{1+2 \lambda}{12}(2 \lambda-1)(\lambda+1)<\frac{(1+2 \lambda)(7+2 \lambda)^{3 / 2}}{36 \sqrt{3\left(9-4 \lambda^{2}\right)}} .
$$

Let now $u \in[0,2)$, and define

$$
t_{w}:=\frac{(2 \lambda+5) u}{4(2-u)}
$$

We consider two cases.

Case 1. Suppose that $t_{w} \geq 1$, i.e., when $u \geq 8 /(9+2 \lambda)$. Then

$$
\begin{aligned}
\psi(u, t) & \leq \psi(u, 1)=\frac{1+2 \lambda}{192}\left[-\left(9-4 \lambda^{2}\right) u^{3}+4(7+2 \lambda) u\right] \\
& \leq \psi\left(u_{0}, 1\right)=\frac{(1+2 \lambda)(7+2 \lambda)^{3 / 2}}{36 \sqrt{3\left(9-4 \lambda^{2}\right)}}
\end{aligned}
$$

where

$$
u_{0}:=2 \sqrt{\frac{2 \lambda+7}{3\left(9-4 \lambda^{2}\right)}} .
$$

Note that $8 /(9+2 \lambda) \leq u_{0}<2$. Indeed, the first inequality is equivalent to $16 \lambda^{3}+364 \lambda^{2}+576 \lambda+135 \geq 0$, and the second to $-12 \lambda^{2}-2 \lambda+20>0$, which are clearly true when $\lambda \in[1 / 2,1]$.

Case 2. Suppose that $t_{w}<1$, i.e., $u<8 /(2 \lambda+9)$. Then

$$
\begin{aligned}
\psi(u, t) & \leq \psi\left(u, t_{w}\right) \\
& =\frac{1+2 \lambda}{1536}\left[9(1+2 \lambda)^{2} u^{3}+2(1+2 \lambda)(9+2 \lambda) u^{2}+128\right] \\
& \leq \frac{1+2 \lambda}{1536}\left[9(1+2 \lambda)^{2}\left(\frac{8}{9+2 \lambda}\right)^{3}+2(1+2 \lambda)(9+2 \lambda)\left(\frac{8}{9+2 \lambda}\right)^{2}\right. \\
& \quad+128] \\
= & \frac{(1+2 \lambda)\left(423+414 \lambda+164 \lambda^{2}+8 \lambda^{3}\right)}{6(9+2 \lambda)^{3}}
\end{aligned}
$$

In view of $(28)$ and $(29)$, it remains to show that for $\lambda \in[1 / 2,1]$

$$
\frac{(1+2 \lambda)\left(423+414 \lambda+164 \lambda^{2}+8 \lambda^{3}\right)}{6(9+2 \lambda)^{3}}<\frac{(1+2 \lambda)(7+2 \lambda)^{3 / 2}}{36 \sqrt{3\left(9-4 \lambda^{2}\right)}},
$$


which is equivalent to

$$
\begin{gathered}
181873107+398886930 \lambda+388014624 \lambda^{2}+219687552 \lambda^{3}+79767072 \lambda^{4} \\
+192585560 \lambda^{5}+3096064 \lambda^{6}+319488 \lambda^{7}+19200 \lambda^{8}+512 \lambda^{9}>0 .
\end{gathered}
$$

To see that the inequality is sharp, let

$$
p(z)=\frac{1-z^{2}}{1-u_{0} z+z^{2}}, \quad z \in \mathbb{D},
$$

with

$$
\begin{aligned}
& p_{1}=2 \sqrt{\frac{2 \lambda+7}{3\left(9-4 \lambda^{2}\right)}}, \quad p_{2}=\frac{2\left(12 \lambda^{2}+4 \lambda-13\right)}{3\left(9-4 \lambda^{2}\right)}, \\
& p_{3}=\frac{72 \lambda^{2}+16 \lambda-106}{3\left(9-4 \lambda^{2}\right)} \sqrt{\frac{2 \lambda+7}{3\left(9-4 \lambda^{2}\right)}}
\end{aligned}
$$

which, on substituting into (27), gives the equality in Theorem 6.1.

Choosing $\lambda=1 / 2$, and $\lambda=1$, we deduce the following sharp inequalities.

Corollary 6.2. If $f \in \mathcal{C}$, then

$$
\left|J_{2,3}(f)\right| \leq \frac{4}{9 \sqrt{3}},
$$

proved in [4], and if $f \in \mathcal{F}_{O}(1)$, then

$$
\left|J_{2,3}(f)\right| \leq \frac{9}{4 \sqrt{15}},
$$

proved in $[8]$.

\section{Acknowledgements}

The authors would like to thank the referee for his constructive comments and suggestions that helped to improve the clarity of this manuscript.

Data Availability Statement Not applicable. The manuscript has no associated data.

Code availability Not applicable.

Funding Not applicable.

\section{Declarations}

Conflicts of interest The authors declare that they have no conflict of interest. 
Open Access. This article is licensed under a Creative Commons Attribution 4.0 International License, which permits use, sharing, adaptation, distribution and reproduction in any medium or format, as long as you give appropriate credit to the original author(s) and the source, provide a link to the Creative Commons licence, and indicate if changes were made. The images or other third party material in this article are included in the article's Creative Commons licence, unless indicated otherwise in a credit line to the material. If material is not included in the article's Creative Commons licence and your intended use is not permitted by statutory regulation or exceeds the permitted use, you will need to obtain permission directly from the copyright holder. To view a copy of this licence, visit http:// creativecommons.org/licenses/by/4.0/.

Publisher's Note Springer Nature remains neutral with regard to jurisdictional claims in published maps and institutional affiliations.

\section{References}

[1] Abu Muhanna, Y., Li, L., Ponnusamy, S.: Extremal problems on the class of convex functions of order -1/2. Arch. Math. (Basel) 103(6), 461-471 (2014)

[2] Ali, Md Firoz, Thomas, D.K., Allu, V.: Toeplitz determinants whose elements are the coefficients of analytic and univalent functions. Bull. Aust. Math. Soc. 97(2), 253-264 (2018)

[3] Allu, V., Thomas, D.K., Tuneski, N.: On Ozaki Close-to-convex functions. Bull. Aust. Math. Soc. 99, 89-100 (2019)

[4] Babalola, K.O.: On $H_{3}(1)$ Hankel determinant for some classes of univalent functions. Inequality Theory Appl 6, 1-7 (2010)

[5] Bansal, D., Maharana, S., Prajpat, J.K.: Third order Hankel determinant for certain univalent functions. J. Korean Math. Soc. 52(6), 1139-1148 (2015)

[6] Brickman, L., Hallenbeck, D.J., MacGregor, T.H., Wilken, D.R.: Convex hulls and extreme points of families of starlike and convex mappings. Trans. Amer. Math. Soc. 185, 413-428 (1973)

[7] Brown, J.E., Tsao, A.: On the Zalcman conjecture for starlike and typically real functions. Math. Z. 191, 467-474 (1986)

[8] Cho, N.E., Kwon, O.S., Lecko, A., Sim, Y.J.: Sharp estimates of generalized Zalcman functional of early coefficients for Ma-Minda type functions. Filomat 32(18), 6267-6280 (2018)

[9] Cudna, K., Kwon, O.S., Lecko, A., Sim, Y.J., Śmiarowska, B.: The second and third-order Hermitian Toeplitz determinants for starlike and convex functions of order $\alpha$. Boletín de la Sociedad Matemàtica Mexicana 26, 361-375 (2020)

[10] Duren, P.L.: Univalent functions. Grundlehren der mathematischen Wissenschaften 259, New York, Berlin, Heidelberg, Tokyo, Springer-Verlag (1983)

[11] Efraimidis, I.: A generalization of Livingston's coefficient inequalities for functions with positive real part. J. Math. Anal. Appl. 435, 369-379 (2016)

[12] Efraimidis, I., Vukotić, D.: On the generalized Zalcman functional for some classes of univalent functions. Math. Nachr. 291(10), 1502-1513 (2018)

[13] Janteng, A., Halim, S., Darus, M.: Hankel determinants for starlike and convex functions. Int. J. Math. Anal. 1(13), 619-625 (2007) 
[14] Kaplan, W.: Close-to-convex schlicht functions. Michigan Math. J. 1, 169-186 (1952)

[15] Krushkal, S.L.: Univalent functions and holomorphic motions. J. Analyse Math. 66, 253-275 (1995)

[16] Krushkal, S.L.: Proof of the Zalcman conjecture for initial coefficients. Georgian Math. J. 17, 663-681 (2010)

[17] Libera, R.J., Złotkiewicz, E.J.: Coefficient bounds for the inverse of a function with derivatives in $\mathcal{P}$. Proc. Am. Math. Soc. 87, 251-257 (1983)

[18] Ma, W.: The Zalcman conjecture for close-to-convex functions. Proc. Am. Math. Soc. 104, 741-744 (1988)

[19] Ma, W.: Generalized Zalcman conjecture for starlike and typically real functions. J. Math. Ana. Appl. 234, 328-339 (1999)

[20] Ozaki, S.: On the theory of multivalent functions. Sci. Rep. Tokyo Bunrika Daigaku 4, 45-87 (1941)

[21] Pfaltzgraff, J.A., Reade, M.O., Umezawa, T.: Sufficient conditions for univalence. Ann. Fac. Sci. Univ. Nat. Zaïre (Kinshasa) Sect. Math.-Phys. 2(2), 211218 (1976)

[22] Robertson, M.S.: On the theory of univalent functions. Ann. Math. (2) 37(2), 374-408 (1936)

[23] Thomas D.K., Tuneski N., Allu, V.: Univalent Functions: A Primer. De Gruyter Studies in Mathematics 69, De Gruyter, Berlin, Boston (2018)

[24] Umezawa, T.: Analytic functions convex in one direction. J. Math. Soc. Japan 4, 194-20 (1952)

Vasudevarao Allu

School of Basic Sciences

Indian Institute of Technology Bhubaneswar

Jatni Road, Argul

Bhubaneswar

Odisha 752050

India

e-mail: avrao@iitbbs.ac.in

Adam Lecko

Department of Complex Analysis, Faculty of Mathematics and Computer Science University of Warmia and Mazury in Olsztyn

ul. Słoneczna 54

10-710 Olsztyn

Poland

e-mail: alecko@matman.uwm.edu.pl

Derek K. Thomas

Department of Mathematics

Swansea University Bay Campus

Swansea SA1 8EN

UK

e-mail: d.k.thomas@swansea.ac.uk

Received: February 11, 2021. 
Revised: February 26, 2021.

Accepted: December 1, 2021. 